\title{
ПОКАЗАТЕЛИ НЕПРЕРЫВНОГО СУТОЧНОГО МОНИТОРИРОВАНИЯ ГЛЮКОЗЫ У ЛИЦ С МОDY 2 ДИАБЕТОМ
}

\author{
${ }^{1}$ Рябец М.В., ${ }^{2}$ Овсянникова А.К., ${ }^{2}$ Рымар О.Д. \\ ${ }^{1}$ Федеральное государственное бюджетное образовательное учреждение высшего образования \\ «Новосибирский государственный медицинский университет» Министерства здравоохранения \\ Российской Федерации, 630091, г. Новосибирск, ул. Красный проспект 52 \\ ${ }^{2}$ Научно-Исследовательский Институт терапии и профилактической медицины-филиал \\ Федерального государственного бюджетного научного учреждения «Федеральный \\ исследовательский центр Институт Цитологии и генетики Сибирского отделения Российской \\ академии наук», 630089, г. Новосибирск, ул Бориса Богатова 175/1
}

\begin{abstract}
Введение. В последнее десятилетие проблема ведения пациентов с сахарным диабетом взрослого типа у молодых (Maturity Onset Diabetes of theYoung - MODY диабет) продолжает оставаться важной темой научных дискуссий. С помощью современных технологий, используя метод непрерывного мониторирования глюкозы (НМГ) и показатели вариабельности гликемии (ВГ), подход к диагностике, контролю гликемического профиля и эффективности назначенного лечения может быть оптимизирован и индивидуализирован.
\end{abstract}

Цель работы: определить показатели вариабельности глюкозы по данным непрерывного мониторинга глюкозы у пациентов молодого возраста с MODY 2.

Материалы и методы. Сформирована выборка из 20 человек в возрасте от 18 до 37 лет с подтвержденным молекулярно-генетическим исследованием MODY 2 для проведения НМГ с использованием портативных систем Medtronic MiniMade. Средняя продолжительность мониторинга составила 5 [3; 7] дней. Данные диагностического исследования были синхронизированы с программным обеспечением Medtronic CareLink®Pro. Для оценки вариабельности гликемии использовались параметры: среднесуточный уровень гликемии (BG, Blood glucose), стандартное отклонение (SD, Standart Deviation), значением M (M - value), индекс длительного повышения гликемии (CONGA, Continuous Overlapping NetGlycemic Action), средняя амплитуда колебаний гликемии (MAGE, Mean Amplitude of Glycemic Excurtion), скорость изменеия уровня глюкозы (MAG, Mean Absolut Glucose, индекс риска гипергликемии (HBGI, Hight Blood Glucose Index), индекс риска гипогликемии (LBG, Low Blood Glucose Index), среднее значение рисков гипо/гипергликемии (ADRR, Average Daily Rick Range). Расчёт индексов вариабельности гликемии проводился с использованием программы «GLINVA».

Результаты: Медиана возраста пациентов при диагностировании гипергликемии составила 28 [10; 36] лет. Совокупность выборки однородна - коэффициент вариации (CV) - 17,0 [11,0; 27,0] \% (N менее $40 \%$ ). Среднесуточный уровень гликемии (BG) составил 7,5 $[5,9 ; 11,9]$ ммоль/л, что еще раз подтверждает умеренную гипергликемию у пациентов с MODY 2. Средняя амплитуда колебаний гликемии и скорость изменения уровня глюкозы находятся в диапазоне нормальных значений и сопоставимы со значениями лиц без СД: MAGE $=2,5[1,5-4,0]$ ммоль/л $(\mathrm{N}=0,0-2,8$ ммоль/л); MAG = 2,0 [1,3-2,4] ммоль/л/ч ( $\mathrm{N}=0,5-2,2$ ммоль/л/ч). У пациентов с MODY 2 риск развития гипергликемии находится в диапазоне нормальных значений, как у лиц с нормогликемией. Повышен индекс LGBI и, вероятно, за счёт него повышен и ADRR, что показывает возможность возникновения гипогликемии у исследуемой группы пациентов. Гипогликемические состояния выявились у $36 \%$ пациентов, из них ночная гипогликемия ни выявлена ни у одного пациента. Уровень гликемии выше целевых значений диагностирован у $81 \%$ пациентов. Индекс риска гипергликемии (HBGI) имеет значение 8,0 [6,0;12,0] y.e. $(\mathrm{N}=0,0-7,7)$.

Выводы: 1. У пациентов с MODY 2 определяется невысокая суточная вариабельность глюкозы. 
2. У лиц с мутациями в гене $G C K$ в $36 \%$ определяются гипогликемии лёгкой степени, ночные гипогликемии не характерны для данной группы пациентов.

3. У большинства пациентов с MODY 2 не достигнуты целевые показатели гликемии, что показывает необходимость проведения им коррекции образа жизни и проводимой терапии.

Ключевые слова: MODY 2, сахарный диабет взрослого типа у молодых, непрерывный мониторинг глюкозы (НМГ), Continuous Glucose Monitoring System (CGMS), вариабельность гликемии (ВГ) 